\title{
ESTRATEGIAS DE INNOVACIÓN PARA EMPRESAS STARTUPS
} INNOVATION STRATEGIES TO STARTUPS ENTERPRISES

\section{Ronalty Oliveira Rocha}

ronaltyrocha@gmail.com

Faculdade Maurício de Nassau (UNINASSAU/Aracaju) - Sergipe - SE, Brasil

ORCID: https://orcid.org/0000-0003-4943-4195

\section{Maria Elena Leon Olave}

mleonolave@gmail.com

Programa de Pós-graduação em Administração/Universidade Federal de Sergipe (UFS) - Sergipe - SE, Brasil.

Programa/Universidade - Estado - Sigla, Brasil

ORCID: https://orcid.org/0000-0002-7367-489

\section{Edward David Moreno Ordonez}

edwdavid@gmail.com

Programa de Pós-graduação em Ciência da Computação/Universidade Federal de Sergipe (UFS) - Sergipe - SE, Brasil.

ORCID: https://orcid.org/0000-0002-4786-9243

\section{Resumen}

La innovación está entre los principales atributos de supervivencia y mejora el desempeño de los negocios. Las empresas startups, conscientes de esta realidad, han introducido innovaciones disruptivas e incrementales en el mercado, para alcanzar mayor número de clientes y obtener éxito empresarial. Así, este artículo, presentado en forma de ensayo teórico, tiene como objetivo proponer un modelo conceptual que integre selección de tipologías y estrategias de innovación dirigidas a aumentar la práctica de innovación en startups, especialmente en el sector de tecnología de la información. Con la implementación del modelo propuesto, las empresas startups pueden obtener ganancias de tiempo en la búsqueda de socios, desarrollo de procesos más coherentes y alineados con las tipologías de innovación implementadas, la adopción de estrategias que contribuyan al desarrollo, implementación y comercialización de innovaciones y construcción de lazos sociales.

Palabras-clave: Estrategias de innovación. Innovación. Tipos de innovación. Startups, Modelo conceptual.

\begin{abstract}
Innovation is among the key attributes of business survival and performance, and startups, aware of this reality, have introduced disruptive and incremental innovations in the marketplace to reach more customers and achieve business success. Thus, this article, presented as a theoretical essay, aims to propose a conceptual model that integrates selection of typologies and innovation strategies aimed at increasing the practice of innovation in startups, especially in information technology startups. Starting from the implementation of the proposed model, startup companies can gain time to seek partners, develop more coherent processes and align with the types of innovation implemented, adopt strategies that contribute to the development, implementation and commercialization of innovations and building social bonds.
\end{abstract}

Keywords: Innovation strategies. Innovation, Innovation typologies. Startups. Conceptual model. 


\section{Introducción}

Con el aumento del uso de las herramientas de tecnología de la información, las organizaciones deben enfrentar la imposición de la innovación, que ha sido indicado como uno de los medios más importantes para mantener la competitividad de las empresas. Es consenso entre diferentes estudiosos que la innovación es un factor relevante para diferenciación, alcance de ventaja competitiva y mejor desempeño empresarial y socioeconómico de las empresas (Stoilov, 2015, Imbuzeiro, 2014, Cropley, Kaufman \& Cropley, 2011).

Al considerar el papel de la innovación como instrumento para el desarrollo económico y el crecimiento empresarial, también es necesario analizar cómo las empresas se han dedicado a la práctica de la innovación como estrategia. Heidenreich y Kramer (2016) explican que las estrategias de innovación se refieren a la selección de los mejores y más adecuados planes organizativos para el desarrollo e introducción de nuevos productos y servicios, así como para la apertura de nuevos mercados.

En este contexto, es importante destacar que la literatura académica categoriza diferentes estrategias para la innovación. Cada tipo de estrategia enfatiza un conjunto distinto de actividades, algunas de ellas están centralizadas en alianzas con clientes (Verleye, 2015), otras defienden la práctica de innovación cerrada, exclusivamente dentro de las organizaciones (Schumpeter, 1988), también existen estrategias que defienden la internacionalización como actividad promotora de la innovación (Ren, Eisingerich \& Tsai, 2015), mientras que otros tipos de estrategias enfatizan la colaboración con diferentes instituciones, como competidores, proveedores e instituciones de enseñanza e investigación (Chesbrough, 2003, 2012), además de las estrategias centradas en las asociaciones con otras organizaciones (Alberti \& Pizzurno, 2017).

Hunt (2013) cita las empresas startups como promotoras de la innovación. Por la definición seguida en este estudio, las empresas startups son aquellas que tienen la innovación en su esencia (Ries, 2012), están orientadas por la tecnología de la información y tienen un gran potencial de crecimiento por la introducción de innovaciones en el mercado (Robehmed, 2013, Shontell, 2014).

Turri y Wagner (2015) identificaron que específicamente las empresas startups de tecnología de información son conductoras de la innovación, pues introducen nuevos productos y servicios, generan nuevos procesos de producción y nuevas técnicas organizacionales (Fernandes, 2015) explorando nuevos segmentos de mercado y atrayendo inversionistas.

Especialmente en América Latina es preponderante el papel económico y de desarrollo tecnológico desempeñado por empresas startups. En este contexto merece destacar el potencial de países como Brasil, Chile y Colombia como ambientes fomentadores de la actividad tecnológica en startups (Gavasa, 2018).

Podemos mencionar como ejemplo, Chile, que cuenta con varias iniciativas de fomento a startups, la principal de ellas es Startup Chile que ofrece inversión inicial (capital para iniciar el negócio) para que empresas extranjeras, consideradas startups, usen el mercado local como plataforma de prueba. Según los últimos datos facilitados por el gobierno chileno, sólo em 2015, el mercado de startups generó más de 1600 empleos y movió más de 100 millones de dólares de capital externo. Otro mercado de relevancia en América Latina es el mercado colombiano, especialmente porque el gobierno mantiene el programa Innpulsa, un programa gubernamental focalizado en el fomento y apoyo a la fase inicial de las startups (Egusa, 2018).

En lo que se refiere a Brasil, se resalta que, de acuerdo con la Asociación Brasileña de Startups (Abstartups, 2017), en 2016 había más de 10.000 mil empresas startups en funcionamiento en el país. Estos negocios movieron más de 10 mil millones de reales en 2016 haciendo de Brasil, el principal ecosistema de staturps de América latina, y el $12^{\circ}$ mercado más prometedor para startups en todo el mundo (Global Startups Ecosystem Report, 2017). 
En general, lo que se percibe es que América Latina, especialmente por las políticas gubernamentales adoptadas, revela para el mundo su potencial para recibir inversiones financieras y promover el desarrollo de empresas startups (Egusa, 2018).

Considerando lo anterior y el potencial para el desarrollo económico y creación de oportunidades de empleo en empresas startups, así como teniendo en cuenta la importancia de una selección adecuada de las estrategias dirigidas hacia la innovación y la búsqueda por la supervivencia de estos negocios, este trabajo tiene como objetivo proponer un modelo teórico que integra la selección de tipos y estrategias de innovación dirigidas a aumentar la práctica innovadora en empresas startups del sector de tecnología de la información.

\section{Innovación: aspectos conceptuales}

Inicialmente es necesario presentar de forma resumida el concepto de innovación. Sobre este tema, Ismail y Abdmajid (2007) nos recuerdan que mientras que la innovación ha alcanzado prominencia en la literatura académica, estudios sobre este tema siguen siendo complejos y fragmentados, ya que los diferentes autores analizan el mismo asunto bajo diferentes posiciones ontológicas y epistemológicas.

Con el fin de democratizar debates sobre el tema se presenta en el Tabla 1 una breve revisión sobre los conceptos de innovación y los autores que los defienden.

Tabla 1 - Conceptos de innovación

\begin{tabular}{|c|c|c|}
\hline Elemento Definidor & Autores & Definición \\
\hline \multirow{3}{*}{$\begin{array}{l}\text { Introducción de } \\
\text { novedades }\end{array}$} & Schumpeter (1939). & $\begin{array}{l}\text { Nuevas combinaciones de recursos ya existentes para } \\
\text { acceder a nuevos mercados, producir nuevos productos, o } \\
\text { producir antiguos de una forma más eficiente. }\end{array}$ \\
\hline & $\begin{array}{l}\text { Manual de Oslo (OCDE, } \\
\text { 2006). }\end{array}$ & $\begin{array}{l}\text { Implementación de un nuevo, o mejorado, producto o } \\
\text { servicio, proceso productivo, método de marketing y/ o } \\
\text { método organizacional en las prácticas de negocios, } \\
\text { organización en el lugar de trabajo y/o relaciones } \\
\text { empresariales. }\end{array}$ \\
\hline & Tidd, Bessant y Pavitt (2008) & $\begin{array}{l}\text { Conjunto de actividades organizacionales para la creación o } \\
\text { adquisición de soluciones, prototipos, intentos de } \\
\text { producción, producción y comercialización de productos y } \\
\text { servicios inéditos o mejorados. }\end{array}$ \\
\hline $\begin{array}{l}\text { Introducción de } \\
\text { novedades y agentes } \\
\text { participantes }\end{array}$ & $\begin{array}{l}\text { Błędów et al (2009), Cropley, } \\
\text { Kaufman y Cropley (2011) }\end{array}$ & $\begin{array}{l}\text { Desarrollo e introducción intencional de ideas nuevas, } \\
\text { útiles y prácticas por parte de individuos, equipos y } \\
\text { organizaciones. }\end{array}$ \\
\hline $\begin{array}{l}\text { Aprendizaje } \\
\text { organizacional }\end{array}$ & $\begin{array}{l}\text { Camisón y - Manfort } \\
\text { Mir (2012) }\end{array}$ & $\begin{array}{l}\text { Resultado visible de la capacidad de las organizaciones para } \\
\text { generar nuevos conocimientos y formas de utilización, } \\
\text { combinación, síntesis y / o mejoras sustanciales para la } \\
\text { introducción de novedades en el mercado. }\end{array}$ \\
\hline Rendimiento & Cropley, Kaufman y & Capacidad de las organizaciones para influir en sus \\
\hline empresarial & $\begin{array}{l}\text { Cropley (2011), Ramadani, Ger } \\
\text { guri (2011), Stoilov (2015). }\end{array}$ & $\begin{array}{l}\text { recursos organizacionales, de marketing, habilidades } \\
\text { gerenciales, conocimientos, capacidades y estrategias } \\
\text { organizacionales para la eficacia y el éxito empresarial. }\end{array}$ \\
\hline $\begin{array}{l}\text { Desempeño } \\
\text { empresarial y } \\
\text { desarrollo }\end{array}$ & Imbuzeiro (2014). & $\begin{array}{l}\text { Conversión de una nueva idea en algo que pueda ser } \\
\text { comercializable o provechoso al progreso de las empresas, } \\
\text { regiones y economías. }\end{array}$ \\
\hline socioeconómico & $\begin{array}{l}\text { Huarng, Ribeiro-Soriano, } \\
\text { (2014). }\end{array}$ & $\begin{array}{l}\text { Además de fomentar la creación de productos y procesos, } \\
\text { la innovación favorece el desempeño de negocios, el } \\
\text { bienestar social y la creación de riquezas en una región o } \\
\text { país. }\end{array}$ \\
\hline
\end{tabular}

Fuente: Elaborado por los autores (2018) 
Como se puede ver el concepto de innovación implica diferentes aspectos, pero todos ellos convergen en la idea de que la innovación es la integración y comercialización de algo nuevo, ya sea un producto, un proceso, un método organizacional final o la práctica de la comercialización, siendo estos, incluso, los principales tipos de innovación (OCDE, 2006).

Es oportuno explicar que las innovaciones de producto, de servicio y de proceso son genéricamente denominadas innovaciones tecnológicas, porque utilizan recursos basados en los conocimientos científicos y tecnológicos desarrollados en universidades, institutos de investigación o en las propias empresas (Silva, Weschenfelder, Esteves, 2014). D 'Alvano e Hidalgo (2012) complementan que las innovaciones de producto, servicio y proceso son de carácter tecnológico por estar insertadas en un conjunto de etapas técnicas, industriales y comerciales que sustentan el lanzamiento exitoso de nuevos productos y servicios o la utilización comercial de nuevos procesos técnicos. En el tabla 2 se describen brevemente los principales tipos de innovación.

Tabla 2 - Tipos de innovación

\begin{tabular}{|c|c|c|}
\hline Autor & Tipología & Descripción \\
\hline $\begin{array}{l}\text { Schumpeter (1988), OCDE } \\
\text { (2006), Akcigit (2010), Imbuzeiro } \\
\text { (2014), Koc y Bozdag (2017). }\end{array}$ & $\begin{array}{l}\text { Innovación radical } \\
\text { Innovación } \\
\text { incremental }\end{array}$ & $\begin{array}{l}\text { Las innovaciones radicales producen cambios } \\
\text { fundamentales en las actividades de una organización } \\
\text { y grandes desvíos en las prácticas existentes, mientras } \\
\text { que las innovaciones incrementales representan un } \\
\text { menor grado de desvío de las prácticas actuales de } \\
\text { una organización. }\end{array}$ \\
\hline $\begin{array}{l}\text { Christensen, Johnson y Rigby } \\
\text { (2002), Besanko et al.,(2010). }\end{array}$ & Innovación disruptiva & $\begin{array}{l}\text { Innovaciones que permiten la entrada de nuevos } \\
\text { participantes en el mercado, a partir de soluciones } \\
\text { simples, que garantizan un rápido crecimiento en el } \\
\text { mercado empresarial. }\end{array}$ \\
\hline $\begin{array}{l}\text { Schumpeter (1988), OCDE } \\
\text { (2006), Silva, Weschenfelder y } \\
\text { Esteves (2014), Heidenreich y }\end{array}$ & $\begin{array}{l}\text { Innovación de } \\
\text { producto / servicio }\end{array}$ & $\begin{array}{l}\text { Introducción de productos / servicios nuevos o } \\
\text { significativamente mejorados en términos de sus } \\
\text { características o usos deseados }\end{array}$ \\
\hline $\begin{array}{l}\text { Kraemer } \\
\text { (2016), Jacobs et al., (2016). } \\
\text { OCDE (2006), Tidd, Bessant y } \\
\text { Pavitt (2008), Forsman } \\
\text { (2011), Ruzzier, Hojnik y } \\
\text { Lipnik (2013), Imbuzeiro (2014). }\end{array}$ & $\begin{array}{l}\text { Innovación de } \\
\text { procesos }\end{array}$ & $\begin{array}{l}\text { Se refieren a los cambios en la forma en que los } \\
\text { productos o servicios se crean y se entregan }\end{array}$ \\
\hline $\begin{array}{l}\text { Kimberly (1981), Evan } \\
\text { (1996), Damapour y Aravind } \\
\text { (2012), Ayhan y Oztemel } \\
\text { (2014), Hamidi y Benabdeljlil, (2015 }\end{array}$ & $\begin{array}{l}\text { Innovación } \\
\text { organizacional / } \\
\text { Gerencial / } \\
\text { Administrativa }\end{array}$ & $\begin{array}{l}\text { Creación e implantación de nuevas prácticas, } \\
\text { procesos, estructuras y/o técnicas de gestión, que } \\
\text { contribuyen a una mayor organización, eficiencia y } \\
\text { desempeño de los negocios. }\end{array}$ \\
\hline $\begin{array}{l}\text { ), Jacobs et al., (2016). } \\
\text { OCDE (2006), Takahashi } \\
\text { (2012), Imbuzeiro (2014). }\end{array}$ & $\begin{array}{l}\text { Innovación de } \\
\text { Marketing }\end{array}$ & $\begin{array}{l}\text { Cambios en la concepción y promoción del producto, } \\
\text { ya sea por cambios significativos en el diseño o } \\
\text { embalaje, posicionamiento, alcance de nuevos } \\
\text { mercados, promoción y precio. }\end{array}$ \\
\hline Tidd, Bessant y Pavitt (2008). & $\begin{array}{l}\text { Innovación de } \\
\text { posición }\end{array}$ & $\begin{array}{l}\text { Introducción de cambios en el contexto empresarial } \\
\text { en que productos y servicios se introducen y se } \\
\text { divulgan en el mercado. }\end{array}$ \\
\hline Tidd, Bessant y Pavitt (2008). & $\begin{array}{l}\text { Innovación de } \\
\text { Paradigma }\end{array}$ & $\begin{array}{l}\text { Cambios en los modelos mentales implícitos que } \\
\text { orientan lo que la empresa hace. }\end{array}$ \\
\hline $\begin{array}{l}\text { Agarwal y Brem (2012), Radjou y } \\
\text { Prabhu (2013), Tiwari, Kalogerakis } \\
\text { y Herstatt (2014). }\end{array}$ & Innovación Frugal & $\begin{array}{l}\text { Producir, rediseñar y desarrollar productos y procesos } \\
\text { a un costo mínimo. }\end{array}$ \\
\hline
\end{tabular}

Fuente: Elaborado por los autores (2018) 
Es importante dejar claro que una innovación puede encuadrarse en diferentes tipos, inclusive existe la posibilidad de combinar diferentes tipos de innovación, lo que provoca en las organizaciones el desarrollo de diferentes capacidades, así como favorece el logro de objetivos empresariales (Damanpour, Walker \& Avellaneda, 2009).

Conociendo el concepto y los tipos de innovación, se hace necesario también debatir las estrategias de innovación consideradas por las organizaciones, especialmente por empresas startups, objeto de estudio en ese ensayo.

\section{Estrategias de Innovación}

La innovación es un imperativo estratégico en las economías actuales (Heidenreich, Kraemer \& 2016), y las estrategias para la innovación se refieren a la selección de los mejores y más adecuados planes de la organización para el desarrollo e introducción de nuevos productos y servicios, así como para la entrada a nuevos mercados (Sanches \& Machado, 2014).

Así, las estrategias para la innovación son enfoques organizacionales para definir los objetivos y la elección de métodos que se utilizarán para potencializar resultados a través de innovaciones (Lendel \& Varmus, 2011). En este tema, Barbosa y Machado (2013) comentan que las estrategias de innovación están distribuidas en diversas clasificaciones, ya que cada autor considera un conjunto distinto de aspectos y parámetros para realizar su tipificación.

Es importante señalar que en la literatura académica aparecen varias estrategias innovadoras como por ejemplo: proactivas y reactivas (Gilbert, 1997), la estrategia de proceso, la estrategia de la velocidad, la estrategia para la innovación, la estrategia de mercado, la estrategia de la tecnología, la estrategia de innovación cuantitativa y aprendizaje (Lynn \& Akgun, 1998), la estrategia de jugar para ganar, la estrategia de jugar para no perder (Dávila, Epstein \& Shelton, 2007), la estrategia defensiva, las estrategias imitativas, la estrategia dependiente, la estrategia tradicional, la estrategia oportunista (Freeman \& Soete, 2008), la estrategia de exploración, la estrategia de explotación (Fauchart \& Keilbach, 2009) y la estrategia de innovación cerrada (Schumpeter, 1988, Love \& Roper, 2015, Hsieh, Lee \& Huang, 2016).

Sin embargo, Doloreux (2013) sostiene que en empresas startups el proceso de innovación ocurre con el auxilio de múltiples funciones, actores y recursos dentro y entre las fronteras de las empresas que forman un conjunto ampliado de diferentes competencias que son fundamentalmente necesarias para transformar las ideas en innovaciones que se demuestren económicamente exitosas (Berne, 2016).

A partir de la comprensión de estos autores, especialmente en lo que se refiere a las diferentes competencias entre las fronteras de las organizaciones que despiertan el proceso de innovación en empresas, este trabajo de investigación prefiere destacar las estrategias de asociaciones con clientes, asociaciones con otras organizaciones, innovación abierta e internacionalización como probables estrategias adoptadas por empresas startups, como puede ser visto en el tabla 3. 
Tabla 3 - Estrategias de innovación

\begin{tabular}{|c|c|c|}
\hline Autores & Estrategia & Descripción \\
\hline $\begin{array}{l}\text { Drechsler y Natter (2012), } \\
\text { Saebi y Foss (2014), Alberti y } \\
\text { Pizzurno (2017). }\end{array}$ & $\begin{array}{l}\text { Asociaciones con otras organizaciones o } \\
\text { asociaciones organizativas }\end{array}$ & $\begin{array}{l}\text { Las organizaciones buscan } \\
\text { conocimientos externos, a través de } \\
\text { alianzas con otras organizaciones, para } \\
\text { adquirir experiencias, evaluar contextos } \\
\text { empresariales e incrementar la cartera } \\
\text { interna de soluciones. }\end{array}$ \\
\hline $\begin{array}{l}\text { Greer y Ley (2012), Seran e } \\
\text { Izvercian (2014), Melo et al., } \\
\text { (2015), Verleye (2015). }\end{array}$ & $\begin{array}{l}\text { Alianzas con clientes, co- } \\
\text { creación, codesarrollo, co-innovación, co- } \\
\text { producción, innovación } \\
\text { colaborativa, usuarios líderes, innovación } \\
\text { participativa y / o innovación centrada en el } \\
\text { usuario: }\end{array}$ & $\begin{array}{l}\text { Esfuerzo conjunto entre la empresa y } \\
\text { los clientes para desarrollar nuevos } \\
\text { productos y servicios por medio de la } \\
\text { comunicación y la interacción. }\end{array}$ \\
\hline $\begin{array}{l}\text { Chesbrough }(2003,2012), \\
\text { Pero-Tur et al, }(2015), \text { Saebi } \\
\text { y Foss (2014), Hsieh, Lee y } \\
\text { Huang (2016), Varichio } \\
(2016) .\end{array}$ & Innovación Abierta & $\begin{array}{l}\text { Búsqueda externa de conocimientos y } \\
\text { colaboración con clientes, proveedores, } \\
\text { competidores e instituciones de } \\
\text { investigación con el fin de combinar } \\
\text { ideas internas con tecnologías externas } \\
\text { para aprovechar el potencial de } \\
\text { novedades en nuevos mercados. }\end{array}$ \\
\hline $\begin{array}{l}\text { García, Avella y Ferna'ndez } \\
\text { (2012), Ren, Eisingerich y } \\
\text { Tsai (2015). }\end{array}$ & Internacionalización & $\begin{array}{l}\text { Las empresas exportadoras pueden } \\
\text { aprender de sus contactos extranjeros, } \\
\text { adoptar nuevas tecnologías de } \\
\text { producción y, consecuentemente, } \\
\text { aumentar la productividad y el } \\
\text { rendimiento organizacional. }\end{array}$ \\
\hline
\end{tabular}

Fuente: Elaborado por los autores (2018)

Con relación a las estrategias para la innovación y de acuerdo con Barbosa y Machado (2013) se percibe que no hay una estrategia mejor o peor, existen estrategias de naturaleza diferenciadas, las cuales son identificadas e implementadas a partir del plan estratégico global de cada organización, una vez que independientemente del tipo, las estrategias de innovación se adoptan con el objetivo de aumentar el desempeño en innovación de la organización o reducir las deficiencias en el desempeño que pueden surgir debido a los cambios en el ambiente empresarial (Carpejani, 2015).

Como se mencionó anteriormente, algunas empresas se destacan por el grande potencial para introducir novedades en el mercado, por ejemplo, las empresas startups. Las empresas startups tienen un potencial reconocido para introducir innovaciones en el mercado, principalmente en el sector de tecnología de la información, pues en los últimos años, estas empresas fueron las principales responsables por encontrar solución a problemas lanzados en el mercado, e introducir innovaciones para atender a las diversas necesidades de los consumidores (Sebrae, 2015). Teniendo en cuenta la importancia de empresas startups para promover la innovación, trataremos de este asunto en la sección siguiente.

\section{Innovación en empresas startups}

Inicialmente se discute que la literatura dispone de amplia conceptualización y diferentes puntos de vista acerca de la definición de startups, incluso el término ha sido utilizado de manera amplia y no muy clara en diferentes contextos (Miranda, Santos Júnior \& Dias, 2016).

Debido a los diferentes enfoques observados en el concepto de empresas startups, la definición adoptada en esta investigación considera los aspectos propuestos por Luger y Koo (2005), así como las definiciones propuestas por Stoilov (2015) y Alberti y Pizzurno (2017). Para Stoilov (2015) las startups son empresas diseñadas para crecer rápidamente, no es necesario trabajar en tecnología o desarrollar 
innovaciones de base tecnológica, sino utilizar la tecnología como una herramienta capaz de potencializar el crecimiento, replicación y escala de esos negocios.

Frente a las diferentes definiciones de startups, los autores de este trabajo entienden que el término startup está relacionado con las empresas que tienen la innovación en su esencia (Ries, 2012), son orientadas por la tecnología de la información y tienen un gran potencial de crecimiento (Robehmed, 2013, Shontell , 2014).

Reforzando ese entendimiento, Hunt (2013) argumenta que las empresas startups desempeñan un papel crucial en los procesos de innovación, pues al inicio de sus actividades, en la llamada fase de idealización, nuevas ideas son introducidas en el mercado y transformadas en estrategias económicamente sostenibles. Por otra parte, la capacidad innovadora de estas empresas es tan expresiva que estos negocios son apuntados como elementos de base para la generación de nuevas ideas para productos y servicios que van de forma rápida a responder las demandas del mercado (Andrade, Lins Filho \& Silva, 2016).

Con relación a este asunto, Andrade, Lins Filho e Silva (2016) evidencian que las startups poseen características especiales para el aprendizaje organizacional y consecuente para aumentar los procesos de innovación. Los autores enfatizan que en startups, la capacidad de aprendizaje está relacionada a la capacidad de innovar de esos negocios, principalmente, debido a que las startups poseen características dirigidas al uso de nuevos modelos de intercambio de conocimiento y comunicación entre empleados, clientes y socios.

Conforme descrito anteriormente la innovación presenta un reconocido potencial para el desarrollo y crecimiento de las empresas, incluso de las clasificadas como startups. A partir de esta constatación y basados en la literatura fueron estructurados seis proposiciones de investigación que son mostradas en el tabla 4.

\section{Tabla 4 - Proposiciones de Investigación}

\begin{tabular}{|c|c|}
\hline Proposiciones & Autores \\
\hline $\begin{array}{l}\text { P1. Empresas startups que direccionan sus estrategias de negocio por la implantación de } \\
\text { innovaciones incrementales estructuradas en la imitación y el perfeccionamiento de productos } \\
\text { existentes garantizan regularidad de ventas y supervivencia empresarial. } \\
\text { P2. Las empresas startups que implementan prácticas estratégicas para la innovación por el uso } \\
\text { de laboratorios internos de investigación especializados en la producción de nuevos } \\
\text { conocimientos y propiedad intelectual para explotación comercial alcanzan mayor grado de } \\
\text { innovación y supervivencia en el mercado. }\end{array}$ & $\begin{array}{l}\text { Chesbrough (2012), Love y } \\
\text { Roper (2015), Stoilov } \\
\text { (2015), Hsieh, Lee y Huang } \\
\text { (2016). }\end{array}$ \\
\hline $\begin{array}{l}\text { P3. El desarrollo de colaboraciones interorganizacionales basadas en la búsqueda de } \\
\text { conocimientos externos, a través de alianzas con otras organizaciones, para adquirir experiencia e } \\
\text { incrementar la cartera interna de soluciones está asociada a un mayor grado de innovación por } \\
\text { startups en el mercado. }\end{array}$ & $\begin{array}{l}\text { Forsman (2011), Drechsler y } \\
\text { Natter (2012), Saebi y Foss } \\
\text { (2014), Alberti y Pizzurno } \\
\text { (2017). }\end{array}$ \\
\hline $\begin{array}{l}\text { P4. La búsqueda externa de conocimientos y colaboración con clientes, proveedores, } \\
\text { competidores e instituciones de investigación con el fin de combinar ideas internas con } \\
\text { tecnologías externas impulsa el potencial para introducción de novedades por startups y puede } \\
\text { hacer estas empresas innovadoras sistémicas. }\end{array}$ & $\begin{array}{l}\text { Pero- tur et al, (2015), Saebi y } \\
\text { Foss (2014), Agrawal, Hasija y } \\
\text { Bhattacharya (2016), Hsieh, Lee } \\
\text { y Huang } \\
\text { (2016), Varichio(2016), Pérez- } \\
\text { Luno et al., (2011). }\end{array}$ \\
\hline $\begin{array}{l}\text { P5. Las asociaciones con clientes promueven el desarrollo de nuevos productos y servicios, así } \\
\text { como la mejoría de los productos / servicios existentes a través de la comunicación e interacción } \\
\text { en las startups, y consecuentemente garantiza mayor nivel de innovación y supervivencia } \\
\text { empresarial a esas empresas. }\end{array}$ & $\begin{array}{l}\text { Belkahla y Triki } \\
\text { (2011), Brunswicker y } \\
\text { Vanhaverbeke (2015), Greer y } \\
\text { Ley (2012), Seran y Izvercian } \\
\text { (2014), Melo et., } \\
\text { al (2015), Verleye (2015). }\end{array}$ \\
\hline $\begin{array}{l}\text { P6. Startups exportadoras pueden aprender con sus contactos extranjeros, a adoptar nuevas } \\
\text { tecnologías de producción y consecuentemente, aumentar la productividad, la práctica de la } \\
\text { innovación y el desempeño organizacional. }\end{array}$ & $\begin{array}{l}\text { Charles y David (2012), Gar'cía, } \\
\text { Avella y } \\
\text { Ferna'ndez (2012), Ren, } \\
\text { Eisingerich y Tsai (2015). }\end{array}$ \\
\hline
\end{tabular}

Fuente: Elaborado por los autores (2018) 
Propuesta de un modelo conceptual para selección e implantación de tipos y estrategias de innovación en startups

Considerando el potencial de las startups de tecnología de la información para la inserción de innovaciones en el mercado y subsidiado en las proposiciones de investigación presentadas, se estructuro una propuesta de modelo conceptual, que contempla tipos y estrategias para integrar el papel de startups como promotoras de la innovación, como aparece en la figura 1.

Figura 1 - Selección e implantación de tipos y estrategias de innovación en empresas Startups

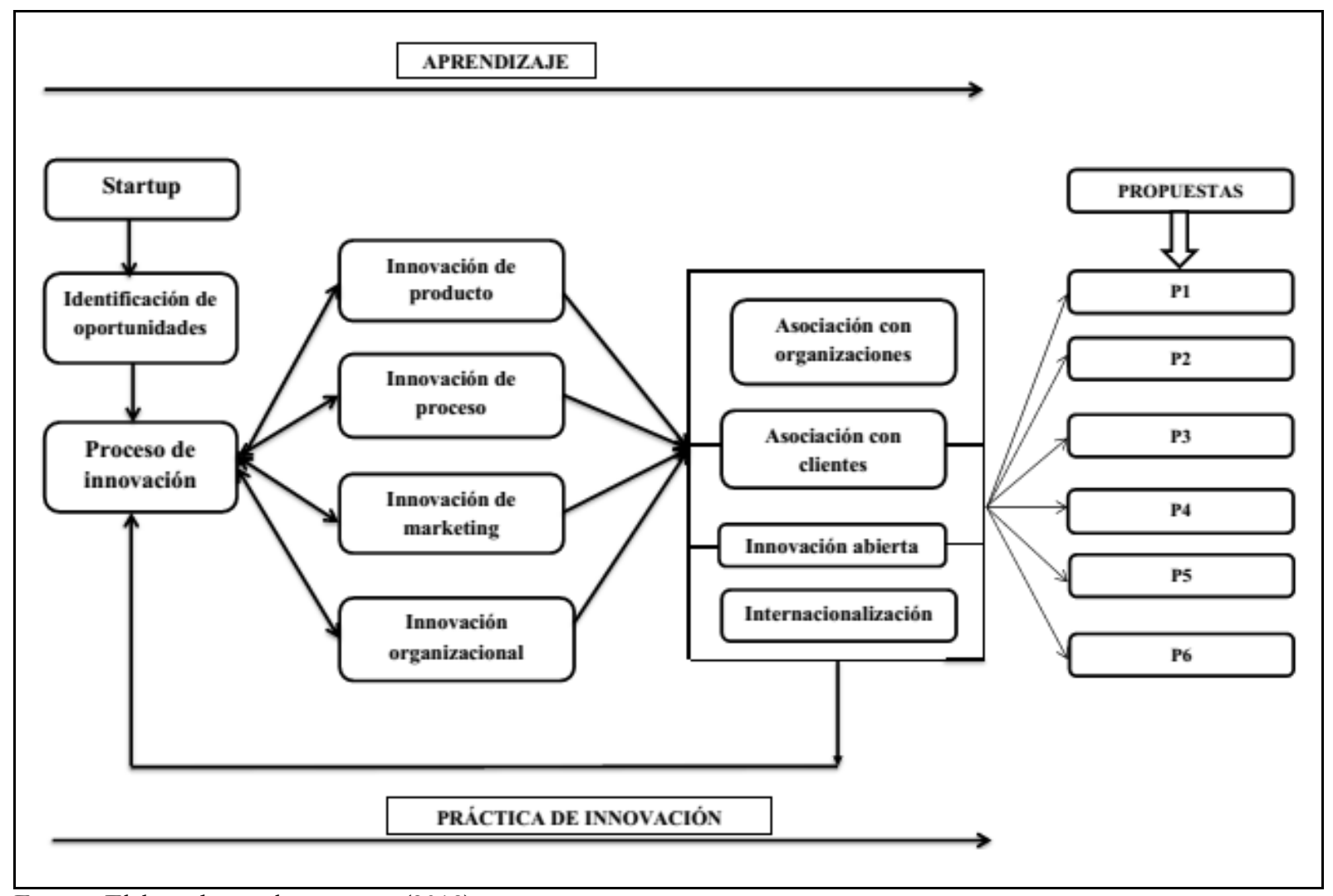

Fuente: Elaborado por los autores (2018)

En el modelo propuesto el proceso de innovación se inicia a partir de la identificación de alguna oportunidad comercial que motiva la introducción de innovaciones por parte de las empresas startups. Se explica que en el modelo propuesto, el proceso de innovación consiste en un conjunto de procedimientos operacionales y gerenciales que favorecen la implantación de una determinada innovación, este proceso no necesariamente necesita estar estructurado en etapas secuenciales, pero debe estar adecuado a la realidad de la empresa que lo implementará, pues el proceso de innovación no es una fórmula establecida, él es estructurado naturalmente por los actores incluidos y / o interesados en su creación (Gollo, 2006).

Después de la identificación de una oportunidad comercial se definen las tipologías de innovación que favorecen la introducción de la innovación proyectada. Como se muestra en el modelo (figura 1) estas innovaciones pueden tener aspectos relacionados con la introducción o mejorías de productos, procesos, prácticas de marketing y/o técnicas de gestión. Es importante subrayar que los tipos de innovación cargan aspectos distintos y que una determinada novedad puede desencadenar diferentes tipos de innovación. La interpretación subyacente es que un tipo determinado de innovación irá prevalecer sobre los otros, lo que no impide la implantación de los demás. De la misma forma que el proceso de innovación, los tipos también son influenciados por la oportunidad identificada y por esa razón influencian y se implantan de acuerdo con el proceso de innovación practicado, es decir, el proceso de innovación dirige la forma como las innovaciones serán implantadas y las tipologías de innovación pueden también promover ajustes en 
el proceso, siendo por esta razón que los tipos y el proceso de innovación están conectados en el modelo, por flechas de doble sentido.

Una vez implantados los tipos más adecuados al negocio, se identifica en cuales actividades del proceso productivo y/o gerencial son necesarios ajustes y mejorías, siendo este el momento en que las empresas startups consideran las estrategias potenciales para adquirir nuevos conocimientos y aumentar la cartera interna de soluciones (Park, Subramaniam \& Stylianou, 2015). Entre las estrategias presentadas en la revisión de la literatura fueron seleccionadas las estrategias de asociación con organizaciones (Saebi \& Foss, 2014), asociación con clientes (Verleye, 2015), innovación abierta (Chesbrough, 2012) e internacionalización (Ren, Eisingerich \& Tsai, 2015). Después de la definición e implantación de la estrategia de innovación que mejor se adecua a las necesidades de las empresas startups, pudiendo inclusive ser más de una entre las varias estrategias propuestas, el proceso se inicia (actualiza), pues el aprendizaje y las técnicas asimiladas pasan a aumentar el conjunto interno de conocimientos de la startup y como consecuencia adiciona cambios en el proceso de innovación.

Como se demuestra en el modelo (figura 1), desde la identificación de oportunidades hasta la definición y práctica de los tipos y estrategias, las startups acumulan conocimientos resultantes de las etapas implantadas, así como aumenta su grado de innovación por sumar prácticas que alteran, ajustan y promueven mejorías en su funcionamiento y posicionamiento de mercado.

Además de los beneficios ya citados, otros beneficios pueden ser proporcionados a las empresas startups a partir de la adopción del modelo propuesto, conforme se presenta en la siguiente sección.

\section{Contribuciones de los hallazgos de la investigación para empresas startups}

Específicamente al funcionamiento empresarial e inserción de novedades por empresas startups, el modelo propuesto evidencia que la selección de tipologías y estrategias de innovación son actividades complementarias e interdependientes entre sí, es decir, la selección de determinada tipología de innovación influye en las estrategias de innovación que pueden se adoptadas. Sobre esta inferencia, es posible añadir que startups que opten por ingresar en el mercado a partir del desarrollo de innovaciones de procesos pueden implementar estrategias del tipo innovación abierta y/o asociación con otras organizaciones, pues de esta forma estos negocios pueden asimilar conocimientos y experiencias de diferentes fuentes académicas y empresariales, así como de negocios ya estructurados en el mercado.

En adición a esta percepción, se explica que las startups que desean ingresar en el mercado a partir de la innovación de producto pueden implementar estrategias de asociación con clientes e innovación abierta, ya que al colaborar y comunicarse directamente con el público consumidor de sus innovaciones, las startups maximizan el potencial de éxito comercial de sus innovaciones, ya que éstas se desarrollarán de acuerdo con las necesidades, expectativas y exigencias del consumidor.

La innovación abierta se destaca por permitir a las empresas startups obtener conocimientos y ayuda (técnico y gerencial) de diferentes instituciones, tales como universidades, proveedores, organismos de investigación y entidades paraestatales (a ejemplo del SEBRAE, SENAI, SENAC y etc.) que estudian y reconocen el comportamiento del cliente. Además, estas instituciones descubren oportunidades comerciales existentes en el mercado y pueden sugerir y orientar la práctica innovadora en empresas startups.

Así, al implementar y desarrollar la innovación abierta, los gestores de startups reducen los riesgos de fracaso comercial (en el lanzamiento de productos/servicios, perfeccionamiento de procesos, adopción de nuevas tácticas de marketing y nuevos métodos para gestión organizacional), así como construyen vínculos sociales sólidos con agentes relevantes del mercado empresarial.

De esta forma, sobresale la recomendación que las empresas startups usen estrategias de acuerdo con las tipologías de innovación implementadas, pues de esta forma, el desarrollo de novedades será realizado 
en mayor conformidad con las perspectivas comerciales de los emprendedores, con mayor alineamiento a las expectativas de los consumidores, y siempre que sea posible, con el apoyo de diferentes agentes del entorno empresarial.

De esta manera, a partir de las inferencias obtenidas con el modelo propuesto, se cree que las empresas startups podrán concebir e implementar innovaciones con ganancias de tiempo en la búsqueda de socios; desarrollo de procesos más coherentes y alineados con las tipologías de innovación implementadas; adopción de estrategias que contribuyan efectivamente al desarrollo, implementación y comercialización de innovaciones; construcción de robustos lazos sociales con agentes empresariales y, no menos importante, fortalecimiento y reconocimiento de la marca de estos negocios.

\section{Consideraciones finales}

Las inferencias obtenidas en ese estudio, aunque de carácter teórico, poseen relevancia para el campo de estudios de innovación y de startups. Inicialmente, el estudio presenta un cuadro resumido sobre la importancia de la innovación para el mantenimiento y la continuidad empresarial de todos los tipos de negocios, sean empresas startups o empresas tradicionales. Además, esta investigación reitera los diferentes tipos de innovación, destacando los principales aspectos de cada tipología, y de esta forma, evidenciando a investigadores y emprendedores las principales características y consecuente aplicación de cada tipo de innovación.

Como se presentó a lo largo de este estudio, las empresas startups son ampliamente reconocidas por su potencial para la inserción de innovaciones en el mercado (HUNT, 2013, SEBRAE, 2015), pero estos negocios no implementan procesos estructurados de desarrollo de innovaciones (GOLLO, 2006), ni siquiera adoptan procedimientos preestablecidos para la selección de tipologías de y/o estrategias de innovación. Por este motivo este artículo, tuvo como objetivo proponer un modelo teórico con la integración de tipologías y estrategias de innovación dirigidas a aumentar la práctica de la innovación en startups, especialmente en startups de tecnología de la información.

En primer lugar, se adoptó el hecho de que la innovación es un concepto ampliamente debatido en el medio académico y empresarial y que, por más que posea diferentes concepciones, la idea central de innovación es la inserción y comercialización de algo nuevo, sea un producto, proceso, método organizacional o práctica de marketing, siendo éstas las principales tipologías de innovación consideradas para esta investigación (y desarrollo del modelo teórico). Además, se ha asumido que startups son empresas que instituyen la innovación como foco, poseen modelos replicables de negocios, están orientadas por el uso de tecnología de la información y poseen gran potencial de crecimiento comercial.

Para complementar el papel de las empresas startups como promotoras de la innovación, este estudio consideró las estrategias de alianzas con otras organizaciones, asociaciones con clientes, innovación abierta e internacionalización como las estrategias más adecuadas para el desarrollo e implementación de innovaciones por estos negocios.

A partir de las consideraciones hechas, esta investigación desarrolló un modelo teórico que integra la selección de tipologías y estrategias de innovación. En realidad, la intención del modelo fue demostrar que la selección de determinada estrategia debe estar alineada a la tipología de innovación implementada por el negocio, una vez que, como se ha discutido anteriormente, existen estrategias más adecuadas y vinculadas a ciertos tipos de innovación, tales como la innovación de procesos y estrategias de asociaciones organizacionales, por ejemplo.

De forma más amplia, el propósito del modelo propuesto es garantizar que las empresas startups puedan obtener beneficios en la selección y implementación de tipologías y estrategias de innovación, beneficios como: ganancias de tiempo en la búsqueda de socios; desarrollo de procesos más coherentes y alineados con las tipologías de innovación implementadas; adopción de estrategias que contribuyan efectivamente 
al desarrollo, implementación y comercialización de innovaciones; construcción de robustos lazos sociales con agentes empresariales y fortalecimiento y reconocimiento de la marca representada por estas empresas.

Otra contribución ofrecida por este estudio son las proposiciones de investigación. Estas proposiciones deben ser objeto de estudios empíricos a fin de validar y/o cambiar las inferencias planteadas en cada proposición.

Lógicamente, que por tratarse de un estudio teórico, esta investigación carece de aplicaciones empíricas que legitimen las conclusiones aquí presentadas. De esta forma, se sugiere que nuevos estudios sean realizados con el objetivo de verificar si la integración entre tipologías y estrategias de innovación efectivamente mejoran los resultados de empresas startups, conforme indicado en el modelo propuesto. Además, nuevos estudios pueden averiguar, en el contexto práctico, si empresas startups adoptan otras estrategias de innovación más allá de las listadas en esa investigación. Por último, nuevos estudios pueden asociar el proceso de innovación desarrollado por empresas startups y la influencia de estos procesos en la selección de tipologías y estrategias de innovación.

\section{Referencias}

Abstartups - Associação Brasileira de Startups, Consultoria Accenture. (2018). O Momento da startup brasileira e o futuro do ecossistema de inovação. Recuperado de < https://abstartups.com.br/PDF/radiografia-startups-brasileiras.pdf>.

Agarwal, N. \& Brem, A. (2012). Frugal and reverse innovation - Literature overview and case study insights from a German MNC in India and China. 18th International Conference on Engineering, Technology and Innovation.

Agrawal, A., Bhattacharya, S. \& Hasija, S. (2016). Cost-Reducing Innovation and the Role of Patent Intermediaries in Increasing Market Efficiency. Production and Operations, Management Society, 25(2), 173-191, 2016.

Akcigit, U. (2010). iFirm Siž, Innovation Dynamics and Growthî, University of Pennsylvania, Working Paper.

Alberti, F. G. \& Pizzurno, E. (2017). Oops, I did it again! Knowledge leaks in open innovation networks with start-ups. European Journal of Innovation Management, 20(1), 50-79.

Andrade, A. P. V., Lins Filho, M. L. \& Silva, G. G. (2017). Capacidade de inovar em startups: Uma abordagem sob a ótica da orientação para a aprendizagem. In: XL Encontro da ANPAD, 2016, Costa do Sauipe. Anais eletrônicos... Costa do Sauípe, 2016. Recuperado de < http://www.anpad.org.br/ anpad/abrir_pdf.php?e=MjE1Mjk= >.

Associação Brasileira de Startups - ABSTARTUPS (2014). Sobre o CASE: O maior eventos para startups da América Latina, 2014. Recuperado de: <https://case.abstartups.com.br/sobre-o-evento-case/>.

Ayhan, M. B. \& Oztemel, E. (2014). A Methodology to Measure the Degree of Managerial Innovation. Journal of Industrial Engineering and Management, 7(1), 153-173.

Barbosa, R. A. \& Machado, A. G. C. (2013). Estratégias de inovação sob a perspectiva da visão baseada em recursos: um estudo na Embrapa. Gestão \& Regionalidade, 29(87), 95-110.

Belkahla, W. \& Triki, A. (2011). Customer knowledge enabled innovation capability: proposing a measurement scale. Journal of knowledge management, 15(4), 648-674.

Belkahla, W. \& Triki, A. (2011). Customer knowledge enabled innovation capability: proposing a measurement scale. Journal of knowledge management, 15(4), 648-674. 
Berne, D. F. (2016). O Grau de Inovação das Indústrias MPE da Região Metropolitana Oeste e Sudoeste de São Paulo. Dissertação (Mestrado em Administração). Faculdades Campo Limpo Paulista. São Paulo.

Besanko, D., Dranove, D., Shanley, M. \& Schaefer, S. (2010). A Economia da Estratégia. Porto Alegre: Bookman.

Besanko, D., Dranove, D., Shanley, M. \& Schaefer, S. (2010). A Economia da Estratégia. Porto Alegre: Bookman.

Bledow, R., Frese, M., Anderson, N., Erez, M., Farr, J. (2009).A dialectic perspective on innovation: Conflicting demands, multiple pathways, and ambidexterity. Industrial and Organizational Psychology, 2, 305337.

Brunswicker, S. \& Vanhaverbeke, W. (2015). Open innovation in small and medium-sized enterprises (SMEs): external knowledge sourcing strategies and internal organizational facilitators. Journal of Small Business Management, 53(4), 1241-1263.

Brunswicker, S. \& Vanhaverbeke, W. (2015). Open innovation in small and medium-sized enterprises (SMEs): external knowledge sourcing strategies and internal organizational facilitators. Journal of Small Business Management, 53(4), 1241-1263.

Camisón, C. \& Monfort-Mir, V. M. (2012). Measuring innovation in tourism from the Schumpeterian and the dynamic-capabilities perspective. Tourism Management, 33, 776 - 789.

Camisón, C. \& Monfort-Mir, V. M. (2012). Measuring innovation in tourism from the Schumpeterian and the dynamic-capabilities perspective. Tourism Management, 33, 776 - 789.

Carpejani, E. (2015). A Influência do Programa ALI no processo de inovação de Micro e Pequenas Empresas do Estado de Sergipe. 2015. 115 f. Dissertação (Mestrado em Administração e Desenvolvimento Empresarial) Universidade Estácio de Sá, Rio de Janeiro.

Charles, R. G. \& David, L. (2012). Collaborative innovation with customers: A review of the literature and suggestions for future research. International Journal of Management Reviews, 14(1), 63-84.

Charles, R. G. \& David, L. (2012). Collaborative innovation with customers: A review of the literature and suggestions for future research. International Journal of Management Reviews, 14(1), 63-84.

Chesbrough, H. (2003). The era of open innovation. MIT Sloan Management Review, 44(3) 35-41.

Chesbrough, H. (2012). Inovação aberta: como criar e lucrar com a tecnologia. Porto Alegre: Bookman, 241 p.

Christensen, C. M. \& Raynor, M.E. (2003). Innovator's solution: creating and sustaining successful. Boston, MA: Harvard Business School Press.

Christensen, C. M., Johnson, M. W. \& Rigby, D. K. (2002). Foundations for growth: how to identify and build disruptive new business. MIT Sloan Management Review, 43(3), 22-31.

Cropley, D. H., Kaufman, J. C. \& Cropley, A. J. (2011). Measuring Creativity for Innovation Management. Journal of Technology Management \& Innovation, 6 (3).

D'alvano, L. \& Hidalgo, A. (2012). Innovation management techniques and development degree of innovation process in service organizations. $R$ and $D$ Management, 42(1).

Damanpour, F. \& Aravind, D. (2012). Managerial Innovation: Conceptions, Processes, and Antecedents. Management and Organization Review, 8(2), 423-454. 
Damanpour, F., Walker, R. M. \& Avellaneda, C. N. (2009). Combinative effects of innovation types and organizational performance: a longitudinal study of service organizations. Journal of Management Studies, 46(4), 650-675.

Davila, T., Epstein, M. J. \& Shelton, R. As regras da inovação - como gerenciar, como medir e como lucrar. Porto Alegre: Bookman, 2007.

Doloreux, D. (2013). What we should know about regional innovation systems of innovation. Technology and Society, 24, 243-263.

Drechsler, W. \& Natter, M. (2012). Understanding a firm's openness decisions in innovation. Journal of Business Research, 65, 438-445.

Drechsler, W. \& Natter, M. (2012). Understanding a firm's openness decisions in innovation. Journal of Business Research, 65, 438-445.

Egusa, C. (2018), Why Startups Are Coming To Latin America: The Allure Of Government-Backed Accelerators, Forbes. Recuperado de: < https://www.forbes.com/sites/forbesagencycouncil/2018/01/11/why-startups-are-coming-to-latinamerica-the-allure-of-government-backed-accelerators/\#27cde6ec7aed $>$.

Fauchart, E. \& Keilbach, M. (2009). Testing a model of exploration and exploitation as innovation strategies. Small Business Economics, 33, 257-272.

Forsman, H. (2011). Innovation capacity and innovation development in small enterprises. A comparison between the manufacturing and service sectors. Research Policy, 40, 739- 750.

Forsman, H. (2011). Innovation capacity and innovation development in small enterprises. A comparison between the manufacturing and service sectors. Research Policy, 40, 739-750.

Freeman, C. \& Soete, L. A economia da inovação industrial. Campinas: Editora da Unicamp, 2008.

García, F., Avella, L. \& Ferna'Ndez, E. (2012). Learning from exporting: The moderating effect of technological capabilities. International Business Review, 26(6), 1099- 1111.

García, F., Avella, L. \& Ferna'Ndez, E. Learning from exporting: The moderating effect of technological capabilities. International Business Review, v. 26, n. 6, p. 1099-1111, 2012.

Gavasa, J. (2018). Best Cities to Create a Startup in Latin America, Panamerican Word, 2018. Recuperado de: <https://www.panamericanworld.com/en/article/best-cities-create-startup-latin-america>.

Gilbert, J. T. (1997). Choosing an inovation strategy, theory and practice. Business Horizons.

Gollo, S.S. (2006). Estratégias de Cooperação Competitiva e a Inovação: O caso da Indicação de Procedência Vale dos Vinhedos - RS. 361 f. Tese (Doutorado em Administração) - Programa de Pós-Graduação em Administração, Universidade Federal do Rio Grande do Sul, Porto Alegre.

Greer, C. R. \& Lei, D. (2012). Collaborative Innovation with Customers: A Review of the Literature and Suggestions for Future Research, International Journal of Management Reviews, v.14(1), 63-84.

Greer, C. R. \& Lei, D. Collaborative Innovation with Customers: A Review of the Literature and Suggestions for Future Research. International Journal of Management Reviews, v.14, n. 1, p.63-84, 2012.

Hamidi, S. \& Benabdeljlil, N. (2015). Managerial and Technological Innovations: Any Relationship? Procedia - Social and Behavioral Sciences, 181, 286 - 292. 
Heidenreich, S. \& Kraemer, T. (2016). Innovations-Doomed to Fail? Investigating Strategies to Overcome Passive Innovation Resistance. Journal of Product Innovation Management, 33(3), 277-297.

Heidenreich, S. \& Kraemer, T. (2016). Innovations-Doomed to Fail? Investigating Strategies to Overcome Passive Innovation Resistance. Journal of Product Innovation Management, 33(3), 277-297.

Hsieh, C.-T., Huang, H.-C. \& Lee, W.-L.(2016). Using transaction cost economics to explain open innovation in start-ups. Management Decision, 54(9), 2133-2156.

Hsieh, C.-T., Lee, W.-L \& Huang, H.-C. (2016). Using transaction cost economics to explain open innovation in start-ups. Management Decision, 54(9), 2133-2156.

Huarng, K. H. \& Ribeiro-Soriano, D.E. (2014). Developmental management: Theories, methods, and applications in entrepreneurship, innovation, and sense making. Journal of Business Research, 67(5), 657662.

Huarng, K. H. \& Ribeiro-Soriano, D.E. (2014). Developmental management: Theories, methods, and applications in entrepreneurship, innovation, and sense making. Journal of Business Research, 67(5) 657-662.

Hunt, R. A. (2013). Entrepreneurial tweaking: an empirical study of technology diffusion through secondary inventions and design modifications by start-ups, European Journal of Innovation Management, 16(2), 148-170.

Imbuzeiro, P. E. (2014). A Proposta e Avaliação de um Modelo da Dinâmica da Inovação nas Micro e Pequenas Empresas Atuando em Redes: Um Estudo do Setor de TIC em Alagoas. 2014, 171 f. Tese (Doutorado em Administração) - Programa de Pós- Graduação em Administração, Universidade Federal de Pernambuco. Recife.

Ismail, W. K. W. \& Abdmajid, R. (2007). Framework of the culture of innovation: a revisit. Journal Kemanusiaan, 9, 38-49.

Jacobs, M., Boersma, L., Dekker, A., Bosmans, G., Merode, F. V, Verhaegen, F., Ruysscher, D., Swart, R., Kengen, C. \& Lambin, P. (2016). What is the degree of innovation routinely implemented in Dutch radiotherapy centres? A multicentre cross-sectional study. BrJ Radiol.

Kimberly, J .R. (1981). Managerial innovation. In P. C. Nystrom, W, Starbuck, H. Handbook of organizational design, New York: Oxford University Press, 84-104.

Koc, T. \& Bozdag, E. (2017). Measuring the degree of novelty of innovation based on Porter's value chain approach. European Journal of Operational Research, 257, 559-567.

Lendel, V. \& Varmus, M. (2011). Creation and implementation of the innovation strategy in the enterprise. Economics and management, 16, 819-825.

Love, J. H. \& Roper, S. (2015). SME innovation, exporting and growth: A review of existing evidence. International Small Business Journal, 33(1), 28-48.

Luger, M. I. \& Koo, J. (2005). Defining and Tracking Businness Strat-Ups. Small Business Economics, 24, $17-28$.

Lynn, S. G. \& Akgun, A. E. (1998). Innovation strategies under uncertainty: A contingency approach for new product development. Engineering Management Journal, 10(3), 11-17.

Mas-Tur, A., Pinazo, P., Tur-Porcar, A. M. \& Sánchez-Masferrer, M. (2015). What to avoid to succeed as an entrepreneu. Journal of Business Research, 68, 2279-2284. 
Melo, C.B. B., Nascimento, J.C. H. B., Melo, M. A., Bernardes J. R. \& Sousa, W. D. (2015). Crowdsourcing como uma ferramenta à inovação estratégica empresarial: uma revisão de literatura. Revista de Empreendedorismo, Inovação e Tecnologia, 1(1), 13-24.

Melo, C.B. B., Nascimento, J.C. H. B., Melo, M. A., Bernardes J. R., \& Sousa, W. D. (2015). Crowdsourcing como uma ferramenta à inovação estratégica empresarial: uma revisão de literatura. Revista de Empreendedorismo, Inovação e Tecnologia, 1(1), 13-24.

Miranda, J. Q., Santos Junior, C. D. \& Dias, A. T. (2016). A influência das variáveis ambientais e organizacionais no desempenho de startups. Revista de Empreendedorismo e Gestão de Pequenas Empresas, 5(1).

Mol, M. J. \& Birkinshaw, J. (2009). The sources of management innovation: When firms introduce new management practices. Journal of Business Research, 1-13.

OECD - Organization for Economic Co-operation and Development, Manual de Oslo - Diretrizes para coleta e interpretação de dados sobre inovação, OECD (2006). - tradução FINEP, Brasília.

Park, S., Stylianou, A., Subramaniam, C. \& Niu, Y. (2015). Information technology and interorganizational learning: An investigation of knowledge exploration and exploitation processes. Information \& Management, 52, 998-1011.

Pérez-Luño, A., Medina, C.C., Lavado, A.C. \& Rodríguez, G.C. (2011). How social capital and knowledge affect innovation, J. Bus. Res, 64(12), 1369-1376.

Pérez-Luño, A., Medina, C.C., Lavado, A.C. \& Rodríguez, G.C. (2011). How social capital and knowledge affect innovation. J. Bus. Res, 64(12), 1369-1376.

Radjou, N. \& Prabhu, J. (2013). Frugal Innovation: A New Business Paradigm. 2013.

Radjou, N. \& Prabhu, J.(2013). Frugal Innovation: A New Business Paradigm. Recuperado de: $<$ http://knowledge.insead.edu/innovation/frugal-innovation-a newbusiness-paradigm-2375>.

Ramadani, V. \& Gerguri, S. (2011). Theoretical Framework of innovation and competitiveness and innovation program in Macedonia. European Journal of Social Sciences, 23(2), 268-276.

Ramadani, V. S. \& Gerguri. Theoretical Framework of innovation and competitiveness and innovation program in Macedonia. European Journal of Social Sciences, 23(2), 268-276.

Ren, S., Eisingerich, A. B. \& Tsai, H.-T. (2015). How do marketing, research and development capabilities, and degree of internationalization synergistically affect the innovation 210 performance of small and medium-sized enterprises (SMEs)? A panel data study of Chinese SMEs. International Business Review, 24, 642-651.

Ren, S., Eisingerich, A. B. \& Tsai, H.-T. (2015). How do marketing, research and development capabilities, and degree of internationalization synergistically affect the innovation performance of small and medium-sized enterprises (SMEs)? A panel data study of Chinese SMEs. International Business Review, 24, 642-651.

Ries, E. (2012). A Startup Enxuta: como os empreendedores atuais utilizam a inovação contínua para criar empresas extremamente bem-sucedidas. Lua de Papel, $1^{\text {a }}$ edição. São Paulo-SP.

Robehmed, N. (2013). What is a Startup? Forbes. Recuperado de: $<$ www.forbes.com/sites/natalierobehmed/2013/12/16/what-is-astartup/\#5c77b0c64c63>. 
Ruzzier, M., Hojnik, J. \& Lipnik, A. (2013). Relationship between innovation and internationalization of slovenian internationalized companies. In: Industry, Science and 211 Police Markers for Sustainable Future, 2013, Slovenia. Recuperado de: <https://ideas.repec.org/h/mgt/micp13/579-595.html>.

Saebi, T. \& Foss, N. J. (2014). Business models for open innovation: matching heterogenous open innovation strategies with business model dimensions. Center for Service InnovationDepartment of Strategy and Management Norwegian School of Economics, $45 \mathrm{f}$.

Saebi, T.\& Foss, N. J. (2014). Business models for open innovation: matching heterogenous open innovation strategies with business model dimensions. Center for Service Innovation-Department of Strategy and Management Norwegian School of Economics, $45 \mathrm{f}$.

Sanches, P. L. B. \& Machado, A. G. C. (2014). Estratégias de inovação sob a perspectiva da ResourcedBased View: análise e evidências em empresas de base tecnológica. Gest. Prod., 21(1), 125-141.

Schumpeter, J. (1939). The Theory of Economic Development. Harvard University Press, Cambridge Massachusetts.

Schumpeter, J.A. (1988). A teoria do desenvolvimento econômico. São Paulo: Nova Cultural, 1988.

Schumpeter,J. A. (1998). Teoria do desenvolvimento econômico: uma investigação sobre lucros, capital, crédito, juro e o ciclo econômico (1 ed., 1934). Tradução de Maria Sílvia Possas. Coleção Os Economistas. São Paulo: Nova Cultural.

Sebrae (2015). Anuário do trabalho na micro e pequena empresa. DIEESE, $4^{\circ}$ ed. São Paulo.

Seran, S. \& Izvercian, M. (2014). Prosumer engagement in innovation strategies The Prosumer Creativity and Focus Model. Management Decision, 52(10), 1968-1980.

Seran, S. \& Izvercian, M. (2014). Prosumer engagement in innovation strategies The Prosumer Creativity and Focus Model. Management Decision, 52, (10), 1968-1980.

Shontell, A. (2014). This is the definitive definition of a startup, Business Insider UK, December. Recuperado de: <http://uk.businessinsider.com/what-is-a-startup-definition-2014-12?r=US\&IR=T>.

Silva, S. M., Weschenfelder, C. F. \& Esteves, P. C. L. (2014). Avaliação da capacidade de inovação das empresas do setor de tecnologia da informação de um município da região do extremo sul de Santa Catarina. In: Seminário de parques tecnológicos e incubadoras de empresa, 2014, Passo Fundo. Anais eletrônicos... Passo Fundo, 2014. Resgatado de: <http://www.anprotec.org.br/Relata/ArtigosCompletos/ID\%2091.pdf >.

Silva, S. M., Weschenfelder, C. F. \& Esteves, P. C. L. (2014). Avaliação da capacidade de inovação das empresas do setor de tecnologia da informação de um município da região do extremo sul de Santa Catarina. In: Seminário de parques tecnológicos e incubadoras de empresa, 2014, Passo Fundo. Anais eletrônicos... Passo Fundo, 2014. Recuperado de: < http://www.anprotec.org.br/Relata/ArtigosCompletos/ID\%2091.pdf >.

Stoilov, I. A. (2015). Innovation in technological start-ups: Korean start-up ecosystem. Universitat Autonoma de Barcelona - Degree: Business Administration and Management.

Tidd, J., Bessant, J. \& Pavitt, K. (2008). Gestão da inovação. Porto Alegre: Bookman.

Tiwari, R., Kalogerakis, K. \& Herstatt, C. (2014). Frugal innovation and analogies: some propositions for product development in emerging economies. Working Paper, n. 84, Hamburg University of Technology, 49,15-23. 
Tiwari, R., Kalogerakis, K. \& Herstatt, C. (2014). Frugal innovation and analogies: some propositions for product development in emerging economies. Working Paper, Hamburg University of Technology, 49, $15-23$.

Turri, S. N. Z. \& Wagner, B. S. (2015). Fatores críticos de sucesso de startups/TI. In: IV Simpósio Internacional de Projetos, Inovação e Sustentabilidade, 2015, São Paulo. Anais... São Paulo, 2015. Recuperado de: < https://singep.org.br/4singep/resultado/280.pdf>.

Varrichio, P. C. (2016). Uma discussão sobre a estratégia de inovação aberta em grandes empresas e os programas de relacionamento voltados para startups no Brasil. RACEF - Revista de Administração, Contabilidade e Economia da Fundace. 7(1), Ed. Esp. Ecossistemas de Inovação e Empreendedorismo, 148-161.

Varrichio, P. C. (2016). Uma discussão sobre a estratégia de inovação aberta em grandes empresas e os programas de relacionamento voltados para startups no Brasil. RACEF, 7(1), 148-161.

Verleye, K. (2015). The co-creation experience from the customer perspective: its measurement and determinants. Journal of Service Management, 26(2), 321-342. 